\section{BETting on OncoFusion}

\section{By Kai-Jye Lou, Senior Writer}

Medivation Inc. has licensed a portfolio of BET bromodomain inhibitors that could have better efficacy than the company's castrationresistant prostate cancer drug Xtandi enzalutamide and could provide the biotech with a new way to disrupt androgen receptor signaling. The company got the molecules from OncoFusion Therapeutics Inc. on the same day the startup's founders published compelling preclinical data for inhibiting BET in the indication. ${ }^{1}$

Under the April 23 deal, Medivation will have exclusive, worldwide rights to selected BET inhibitors. In return, OncoFusion is eligible to receive undisclosed up-front payments and milestones plus royalties.

Also that day, a University of Michigan Medical School team led by OncoFusion cofounder Arul Chinnaiyan published a study in Nature identifying a previously unknown molecular interaction between BET

"What makes this particular
story interesting is the
characterization and
mechanistic analysis of this
molecular pathway showing
how BET is involved in the
transcription of AR-regulated
genes." -Robert Sims,
Constellation Pharmaceuticals Inc.

and mechanistic analysis of this molecular pathway showing how BET is involved in the transcription of AR-regulated genes," said Robert Sims, director of biology at Constellation Pharmaceuticals Inc.

Constellation's lead BET bromodomain inhibitor, CPI-0610, is in a Phase I trial to treat progressive lymphomas.

"Based on the proposed mechanism, the advantage of using BET inhibitors compared to current AR-based therapies is targeting AR-dependent signaling from two angles-the recruitment of AR to DNA as well as recruitment of BRD4 [bromodomain containing 4] and transcriptional machinery to AR-BRD4-occupied genes," wrote Olena Barbash in an e-mail to $S c i B X$.

Barbash is an investigator in oncology R\&D at GlaxoSmithKline plc and a coauthor on a study published in 2013 demonstrating the ability of GSK525762 to decrease MYC expression as well as cell proliferation and tumor burden in prostate cancer models. ${ }^{2}$

"The data published in the study are quite robust, and we are clearly excited about the results," said Medivation president and CEO David Hung. "We've also had the opportunity to look at some additional unpublished data showing that bromodomain inhibition represents a very interesting therapeutic approach that warrants the investment."

Hung said that JQ1's dearth of effects on normal prostate tissues suggests the potential to use BET bromodomain inhibitors in patients with earlier stage disease. He declined to say what specific indications Medivation plans to pursue with the BET inhibitors.

GlaxoSmithKline's GSK525762 is in Phase I testing to treat NUT midline carcinoma or other epithelial cancers. The pharma was not involved in the Nature study and is not disclosing whether it has plans to develop GSK525762 in CRPC.

Chinnaiyan is a professor of pathology and urology at the medical school and an investigator at the Howard Hughes Medical Institute.

In $\mathrm{AR}^{+}$human CRPC cell lines, the research tool BET bromodomain inhibitor JQ1 caused more potent suppression of AR-mediated gene transcription than Xtandi, which is a small molecule AR antagonist.

In addition to blocking AR-mediated gene transcription, JQ1 also inhibited the activity and expression of two known oncogenes: $v$-ets erythroblastosis virus E26 oncogene homolog (ERG) and c-Myc (MYC).

Importantly, Chinnaiyan's group replicated the in vitro results with a clinical-stage BET bromodomain inhibitor called GSK525762 (formerly I-BET762).

In mice with metastatic CRPC, JQ1 decreased tumor volume and weight compared with Xtandi.

Bromodomain inhibition also showed a safety advantage. Healthy mice treated with Xtandi for 30 days developed abnormal prostate morphology and showed shrinkage of prostate tissues, whereas those treated with JQ1 or vehicle did not.

Chinnaiyan noted that OncoFusion's own BET inhibitors also have been tested and have shown similar or better results than those reported in the Nature paper, but he declined to provide details.

"What makes this particular story interesting is the characterization

\section{BETting on safety, against resistance}

Despite being labeled castration resistant, CRPC cells often remain dependent on androgen signaling for their survival, and multiple studies have shown that the transition to castration-resistant disease is associated with reactivation of signaling pathways that involve the $\mathrm{AR} .{ }^{3,4}$

Xtandi is one of two approved drugs for CRPC that work by restoring the blockade of androgen signaling after standard androgen deprivation therapies have failed. The other is Johnson \& Johnson's Zytiga abiraterone acetate.

In its 4Q13 earnings report, Medivation said that partner Astellas Pharma Inc. reported $\$ 161.9$ million in global sales for Xtandi, up $282 \%$ from $\$ 57.4$ million in the same period in 2012 . The biotech reports its 1Q14 sales figures this week.

For 1Q14, J\&J reported $\$ 512$ million in global sales of Zytiga, up $49 \%$ from $\$ 344$ million in the same period in 2013 . Last June, the pharma augmented its prostate cancer pipeline with its acquisition of Aragon Pharmaceuticals Inc. for $\$ 650$ million in cash up front plus up to $\$ 350$ million in milestones. The deal gave the pharma ARN-509, a direct AR antagonist that is in Phase II testing to treat CRPC. ${ }^{5}$ 
Figure 1. Blocking androgen signaling in castration-resistant prostate cancer. Nearly all pathways associated with the transition from androgen-dependent to castration-resistant prostate cancer (CRPC) include reactivation of signaling pathways that involve the androgen receptor (AR). In prostate cancer cells, testosterone-bound ARs form dimers that translocate into the nucleus, in which they get recruited to target gene loci on DNA along with an RNA polymerase to initiate the transcription of AR-regulated genes.

At least two targeted therapies are marketed to treat CRPC that can block androgen signaling after first-line androgen ablation therapies are no longer effective. The more upstream-targeted drug is Johnson \& Johnson's Zytiga abiraterone acetate (green circle), a small molecule inhibitor of cytochrome P450 $17 \alpha$-hydroxylase/C17, 20 lyase (CYP17) [a]. The lyase is responsible for converting testosterone precursors such as dehydroepiandrosterone (DHEA) into testosterone, so inhibiting its function decreases the amount of testosterone available to bind to ARs.

The other drug is Medivation Inc.'s

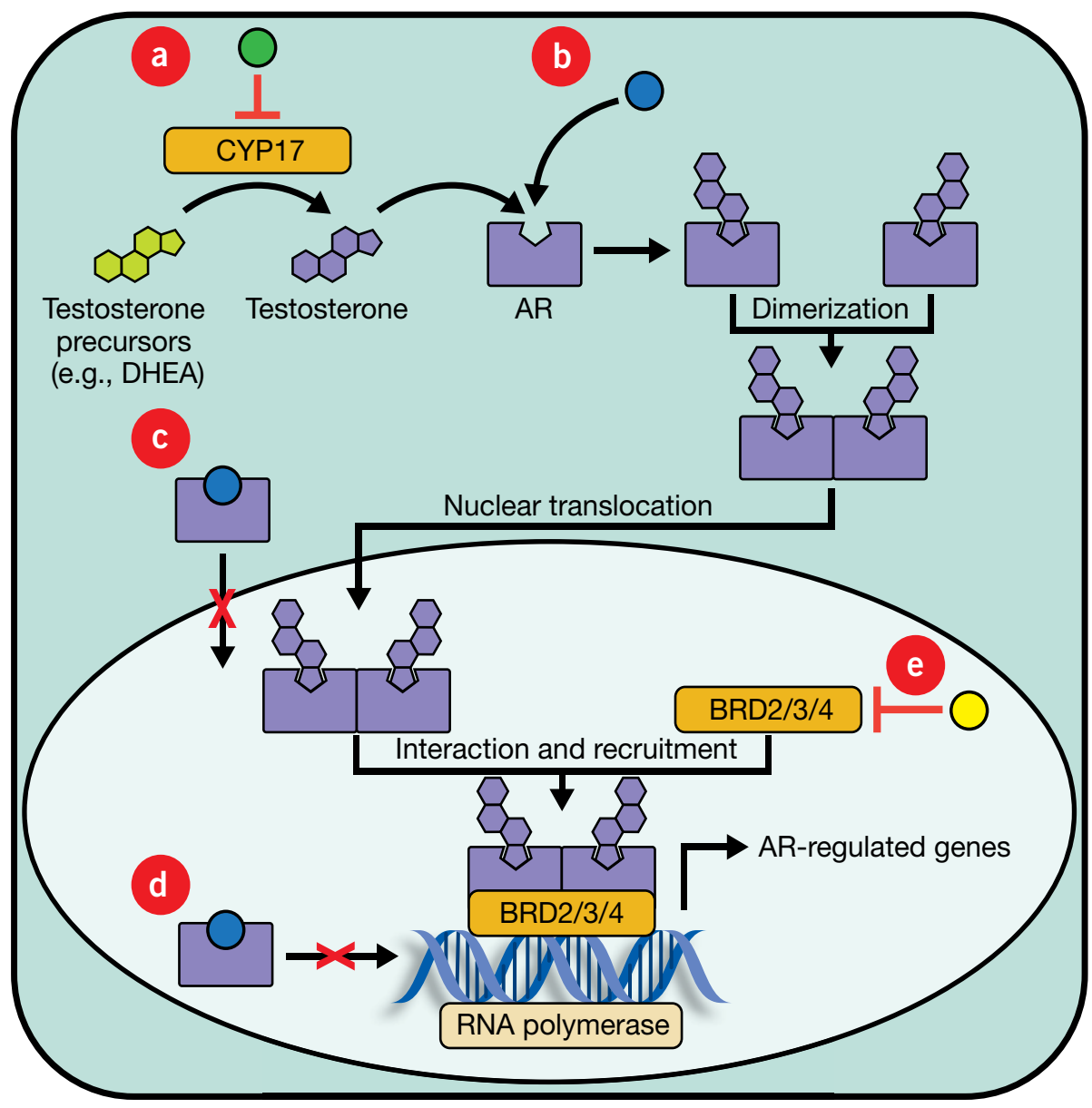
Xtandi enzalutamide (blue circle). The drug blocks AR signaling by competing with testosterone for binding to AR [b], which inhibits the translocation of AR into the nucleus [c] and prevents recruitment of nuclear AR to DNA [d].

As reported in Asangani et al., researchers identified a new molecular interaction between AR and BET bromodomain-containing proteins such as BRD2, BRD3 and BRD4. The group found that blocking the AR and BRD2/3/4 interactions with the BET bromodomain inhibitor JQ1 (yellow circle) blocks the co-recruitment of AR and the BET bromodomain-containing proteins to DNA [e]. This results in decreased expression of multiple AR-regulated genes and also inhibits the activity of two known oncogenes: $v$-ets erythroblastosis virus E26 oncogene homolog (ERG) and c-Myc (MYC).

Zytiga blocks androgen synthesis by inhibiting cytochrome $\mathrm{P} 450$ $17 \alpha$-hydroxylase/C17, 20 lyase (CYP17). The lyase converts androgen precursors into testosterone. Xtandi is a direct AR antagonist.

Not all patients respond to the drugs, and resistance mechanisms have already been reported. ${ }^{6-9}$

"The promise of BET inhibitors in prostate cancer is that they could potentially have activity in cases where resistance has developed against current antiandrogen therapies," said Chinnaiyan. "One could also imagine combining enzalutamide with a BET inhibitor to induce more complete squelching of androgen receptor signaling."

Barbash noted that "combinations of BET inhibitors with current therapies in CRPC as well as novel targeted agents could offer greater efficacy or resensitization of resistant tumors to current agents."

BET bromodomain inhibitors would work downstream of Zytiga and Xtandi by blocking the recruitment of AR to BET bromodomain proteins, which would in turn inhibit the transcription of AR-regulated genes (see Figure 1, "Blocking androgen signaling in castrationresistant prostate cancer").

"They show that these BET inhibitors could induce tumor growth inhibition and tumor shrinkage, but what we don't know at this time is how long the effect could last," said Howard Scher, chief of the genitourinary oncology service and chair in urologic oncology at the Memorial Sloan-Kettering Cancer Center. "We also don't know what changes would be associated with resistance to these compounds." He also cautioned that as the prostate cancer space becomes more crowded, it will get more difficult for newer drug candidates to show improvements in survival over standard of care.

Barbash and GlaxoSmithKline's Anastasia Wyce both noted that studies to evaluate potential therapeutic combinations with BET bromodomain in CRPC models will help guide further development of these compounds in the prostate cancer space. Wyce is an investigator in biology at the pharma. 


\section{ANALYSIS}

Chinnaiyan said that his group at the University of Michigan Medical School is working out additional details of the activity of BET bromodomain inhibitors in prostate as well as in other cancers. He said that the work could aid the development of companion diagnostics to identify patients who would be responsive to BET bromodomain inhibitors.

The University of Michigan has filed a patent application covering the composition of matter of its BET bromodomain inhibitors. The IP has been to OncoFusion.

\section{Lou, K.-J. SciBX 7(18); doi:10.1038/scibx.2014.510}

Published online May 8, 2014

\section{REFERENCES}

1. Asangani, I.A. et al. Nature; published online April 23, 2014; doi:10.1038/nature13229

Contact: Arul M. Chinnaiyan, University of Michigan Medical School, Ann Arbor, Mich. e-mail: arul@umich.edu

2. Wyce, A. et al. Oncotarget 4, 2419-2429 (2013)
3. Attar, R.M. et al. Clin. Cancer Res. 15, 3251-3255 (2009)

4. Dutt, S.S. \& Gao, A.C. Future Oncol. 5, 1403-1413 (2009)

5. Cukier-Meisner, E. BioCentury 21(25), A7-A9; June 24, 2013

6. Balbas, M.D. et al. eLife 2, e00499; published online April 9, 2013; doi:10.7554/eLife.00499

7. Lou, K.-J. SciBX 6(18); doi:10.1038/scibx.2013.429

8. Li, Y. et al. Cancer Res. 73, 483-489 (2013)

9. Arora, V.K. et al. Cell 155, 1309-1322 (2013)

\section{COMPANIES AND INSTITUTIONS MENTIONED}

Astellas Pharma Inc. (Tokyo:4503), Tokyo, Japan Constellation Pharmaceuticals Inc., Cambridge, Mass. GlaxoSmithKline plc (LSE:GSK; NYSE:GSK), London, U.K Howard Hughes Medical Institute, Chevy Chase, Md. Johnson \& Johnson (NYSE:JNJ), New Brunswick, N.J. Medivation Inc. (NASDAQ:MDVN), San Francisco, Calif. Memorial Sloan-Kettering Cancer Center, New York, N.Y. OncoFusion Therapeutics Inc., Ann Arbor, Mich.

University of Michigan, Ann Arbor, Mich.

University of Michigan Medical School, Ann Arbor, Mich. 